\title{
Detection of Fusarium oxysporum f. sp. fragariae from Infected Strawberry Plants
}

\author{
Alyssa Burkhardt, ${ }^{1}$ Peter M. Henry, ${ }^{2}$ Steven T. Koike, ${ }^{3}$ Thomas R. Gordon, ${ }^{2}$ and Frank Martin ${ }^{1, \dagger}$ \\ ${ }^{1}$ Crop Improvement and Protection Research Unit, U.S. Department of Agriculture Agricultural Research Service, Salinas, CA \\ 93905; ${ }^{2}$ Plant Pathology Department, University of California, Davis, CA 95616; and ${ }^{3}$ TriCal Diagnostics, Hollister, CA 95023
}

\begin{abstract}
Isolates of the Fusarium oxysporum species complex have been characterized as plant pathogens that commonly cause vascular wilt, stunting, and yellowing of the leaves in a variety of hosts. F. oxysporum species complex isolates have been grouped into formae speciales based on their ability to cause disease on a specific host. $F$. oxysporum f. sp. fragariae is the causal agent of Fusarium wilt of strawberry and has become a threat to production as fumigation practices have changed in California. F. oxysporum f. sp. fragariae is polyphyletic and limited genetic markers are available for its detection.

In this study, next-generation sequencing and comparative genomics were used to identify a unique genetic locus that can detect all of the somatic compatibility groups of $F$. oxysporum $\mathrm{f}$. sp. fragariae identified in California. This locus was used to develop a TaqMan quantitative polymerase chain reaction assay and an isothermal recombinase polymerase amplification (RPA) assay that have very high sensitivity and specificity for more than 180 different isolates of the pathogen tested. RPA assay results from multiple field samples were validated with pathogenicity tests of recovered isolates.
\end{abstract}

Fusarium oxysporum species complex is a diverse group of filamentous, ascomycetous fungi that are soilborne and able to cause disease in many economically important crop species (Gordon 2017; Ma et al. 2013; Michielse and Rep 2009). F. oxysporum is commonly referred to as a species complex because it includes many clonally reproducing lineages (Michielse and Rep 2009). Symptoms commonly associated with F. oxysporum include wilting, stunting, yellowing, and eventual death of the plant (Michielse and Rep 2009). In addition to a wide range of pathogenic isolates, the majority of clonal lineages in the F. oxysporum species complex are considered to be nonpathogenic and persist by causing asymptomatic root infections and/or living saprophytically (Gordon 2017; Michielse and Rep 2009). When members of the $F$. oxysporum species complex are isolated from a host, it is typically necessary to perform pathogenicity tests to confidently determine whether a given isolate is pathogenic on that particular host (O'Donnell et al. 2009 ). Within the $F$. oxysporum species complex, more than 100 subdivisions of host-specific strains have been designated as formae speciales based on host range, but this designation does not necessarily reflect phylogenetic similarity (Gordon 2017; Michielse and Rep 2009; O'Donnell et al. 2009). Although some formae speciales are composed of a single somatic compatibility group, many have been found to include multiple somatic compatibility groups and appear to be polyphyletic

${ }^{\dagger}$ Corresponding author: F. Martin; Frank.Martin@ars.usda.gov

Funding: The authors gratefully acknowledge funding from the U.S. Department of Agriculture California Department of Food and Agriculture Specialty Crop Block Grant Program (grant SCB14052) and the California Strawberry Commission that supported this work.

*The $\boldsymbol{e}$-Xtra logo stands for "electronic extra" and indicates that one supplementary figure and one supplementary table are published online.

The author(s) declare no conflict of interest.

Accepted for publication 10 October 2018.

This article is in the public domain and not copyrightable. It may be freely reprinted with customary crediting of the source. The American Phytopathological Society, 2019.
(Gordon 2017; Michielse and Rep 2009; O'Donnell et al. 2009). These complex evolutionary relationships pose significant challenges for molecular identification of formae speciales.

Despite the challenges of attempting to group asexually reproducing members of the $F$. oxysporum species complex into distinct phylogenetic groupings, several attempts have been made to clarify the phylogeny of this pathogen using a particular genomic region or a combination of several genomic sequences. Recognizing the challenges of identifying Fusarium species by morphology alone and the confusion of data caused by unclear host designations, Geiser et al. (2004) created FUSARIUM-ID, a database utilizing the translation elongation factor 1-alpha $(E F-1 \alpha)$, which is particularly helpful for determination of Fusarium species but is not always effective at the forma specialis level of classification (Geiser et al. 2004). O'Donnell et al. (2009) used two loci, a partial elongation factor $E F-1 \alpha$ gene sequence and a region of the nuclear ribosomal DNA intergenic spacer region (IGS), to create a phylogeny consisting of $850 \mathrm{~F}$. oxysporum species complex isolates in which 256 two-locus sequence types were identified. However, this two-locus sequencing type can have limited utility in determining the forma specialis of a given isolate based only on its sequencing type, as many formae speciales were found within multiple sequencing types and multiple sequencing types included more than one forma specialis (O'Donnell et al. 2009). It was discovered that many pathogenicity-related genes are encoded on mobile pathogenicity chromosomes that can be exchanged through horizontal gene transfer (Ma et al. 2010; van Dam et al. 2017). Considering this aspect of $F$. oxysporum species complex biology, approaches to genetically classify $F$. oxysporum isolates by forma specialis have used the presence or absence of effectors, such as those coded for secreted in xylem (SIX) genes (Ma et al. 2010; van Dam et al. 2016, 2018). Future studies using more genes and clear pathogenicity tests are needed to further understand the phylogeny and formae speciales designations of many groups of F. oxysporum species complex isolates, including those for which groups of effectors have not yet been identified.

$F$. oxysporum f. sp. fragariae is an example of a forma specialis with a complicated phylogeny and a poorly defined effector profile. This pathogen causes wilt of strawberry, infecting the roots and entering the xylem, causing systemic wilt (Henry et al. 2017). F. oxysporum f. sp. fragariae has been found in Australia, Japan, and in 
other places around the world since the 1960s but has become a problem in California as the use of methyl bromide as a fumigant has been phased out (Henry et al. 2017; Koike et al. 2009). As a result, the $\$ 1.8$ billion strawberry industry in California comprising more than 15,000 hectares is now at risk from Fusarium wilt. Because no treatment exists for protecting strawberries after they have become infected, it is essential to monitor fields for inoculum density of $F$. oxysporum f. sp. fragariae in order to assess the risk of disease and to take appropriate preventative measures like using resistant cultivars in fields with a history of Fusarium wilt (Koike and Gordon 2015; Pincot et al. 2018).

Previous studies have designed assays to detect $F$. oxysporum f. sp. fragariae but developing assays that are specific and rapid has been challenging, given the nature of $F$. oxysporum as a pathogen. In Japan, Suga et al. (2013) developed polymerase chain reaction (PCR) primers (Fofra forward $[\mathrm{F}]$ and reverse $[R])$ to identify this pathogen using a genomic region between two transposable elements. This marker detected $73 F$. oxysporum f. sp. fragariae isolates as true positives and six $F$. oxysporum isolates as false positives; it did not detect 58 nonpathogenic $F$. oxysporum strains or five isolates of other $F$. oxysporum formae speciales (Suga et al. 2013). When this marker was tested on $F$. oxysporum f. sp. fragariae isolates that were collected in California, it did not detect any isolates belonging to somatic compatibility group (SCG) 2 or $5 \%$ of the isolates in SCG1; in total, $\sim 93 \%$ of the pathogenic isolates collected from strawberries in California were detected with the Fofra F/R primers (Henry et al. 2017).

Given these results, an improved marker is needed to detect all of the SCGs in California and to be compatible with in-field diagnostics. In this study, our goals were (i) to use comparative genomics to identify a locus that was specific for all isolates of $F$. oxysporum f. sp. fragariae in California and (ii) to develop a rapid diagnostic assay to detect $F$. oxysporum f. sp. fragariae in infected strawberry plants.

\section{Materials and Methods}

Isolate collection and DNA extraction. Cultures of $F$. oxysporum were obtained from various infected hosts collected from California and Arizona and were grown in potato dextrose broth at $25^{\circ} \mathrm{C}$ for 4 days, with a 10 -s blend on the second day. Fungal tissue was removed from the medium, rinsed with sterile water, blotted to remove excess liquid, and flash-frozen in liquid nitrogen. After lyophilization, DNA for sequencing was extracted from 10 to $15 \mathrm{mg}$ of lyophilized tissue. Some DNA for assay development was extracted from blocks of potato dextrose agar colonized with $F$. oxysporum hyphae. DNA was extracted using either the DNeasy Plant Mini Kit (Qiagen, Redwood City, CA) or the NucleoSpin Plant II Kit (Macherey-Nagel, Düren, Germany). For both kits, an extended incubation time of up to $1 \mathrm{~h}$ was used during the lysis step to increase the overall DNA yield. When using the Macherey-Nagel kit, the following volumes of buffers were used instead of the volumes recommended in the manual for genomic DNA extraction from plants: $600 \mu \mathrm{l}$ of PL1, $625 \mu \mathrm{l}$ of PC, and $600 \mu \mathrm{l}$ of PW1. All DNA was quantified with the Qubit Fluorometer with the Quant-iT dsDNA HS Assay Kit (Invitrogen, Carlsbad, CA). Cultures were stored on dry autoclaved toothpicks colonized by the pathogen and placed in sealed vials kept at room temperature and/or on agar slants. A full list of Fusarium isolates from which DNA was extracted, as well as DNA received from isolates collected in other states and countries, can be found in Supplementary Table S1.

Sequencing the isolates with Illumina. Isolates selected for sequencing were obtained from all of the SCG groups found on strawberry plants, single spored, and phenotyped. Five isolates, 15-7 (GL1551), 15-8 (GL1552), 15-9 (GL1704), 15-10 (GL1702), and 15-11 (GL1703), with the Gordon laboratory reference numbers in parentheses, were nonpathogenic on strawberry. Five isolates, 14-1 (SCG1, GL945), 14-2 (SCG3, GL1315), 14-3 (SCG2, GL 1381), 15-1 (SCG2, GL1059), and 15-6 (SCG1, CL1385), with the SCG groups and Gordon laboratory reference numbers in parentheses, were pathogenic on strawberry. Two of these, 14-1 and 14-2, were amplified with the locus published by Suga et al. (2013), and the remaining three were not amplified by this locus. These pathogenic isolates that were not detected with the previously published locus of Suga et al. (2013) were chosen for Illumina sequencing to find a locus that could identify all of the SCGs reported by Henry et al. (2017). The DNAs were submitted to the DNA Technologies Core of the University of California, Davis Genome Center for 150-bp paired-end HiSeq sequencing and $\sim 70$ million reads per isolate were generated. These reads were analyzed using CLC Genomics Workbench version 9 (Qiagen) using a custom workflow that was executed in two steps. During the first step, reads from the isolates nonpathogenic on strawberry as well as reads from various formae speciales of F. oxysporum deposited in the National Center for Biotechnology Information (NCBI) short read archive (SRX081465, SRX081510, SRX081503, SRX081507, SRX081504, SRX081461, SRX181888, SRX573195, SRR038655, SRX142856, SRX081508, and SRX081464) were mapped to a reference genome, which was a draft PacBio-based genome of $F$. oxysporum $\mathrm{f}$. sp. fragariae isolate 15-2 (GL1080) provided by the Gordon laboratory. The read mapping parameters were set to the following within the workflow: 0.8 similarity fraction, 0.5 length fraction, no masking, length score $=1$, mismatch $\operatorname{cost}=2$, linear gap cost, insertion cost $=3$, deletion cost $=3$, and no global alignment. The reads track from the map reads to reference function was then used to create a mapping graph and to identify graph threshold areas using a window size of 500 and a threshold of 0 reads to identify regions of the pathogenic $F$. oxysporum $\mathrm{f}$. sp. fragariae genome where no reads mapped from nonpathogenic isolates or from other formae speciales of $F$. oxysporum. The first step in this workflow identified a total of 253 regions, which were then used as the input for the second step of the workflow in which all of the reads from the $F$. oxysporum f. sp. fragariae isolates were mapped to these regions using the same parameters described above; however, in this situation, the goal was to identify regions in the reference genome that had a high coverage of reads from pathogenic isolates. As a result, when the steps of creating a mapping graph and identifying graph threshold areas were performed, a window size of 500 was used with a lower read depth threshold of 500 to only select regions that had a high coverage of reads mapping from the pool of pathogenic reads. Using this approach, 82 candidate loci were identified. A draft assembly of each sequenced isolate was created within CLC Workbench using the default de novo assembly parameters and a custom Basic Local Alignment Search Tool (BLAST) database was created from each list of contigs using the BLAST within Workbench. To ensure that the full-length locus was present and to evaluate the copy number in all of the isolates of $F$. oxysporum f. sp. fragariae, the locus was searched against this genome database of the assembly of each isolate using BLASTN (Altschul et al. 1990). To reduce the potential risk of off-target detection, candidate loci were also searched against the NCBI nonredundant DNA database within NCBI using BLASTN to ensure that the loci were not present in any other species.

In addition to the isolates that were sequenced to find the main locus used for detection, additional isolates were later sequenced to better understand the genetic composition and identity of some isolates. These isolates were grown, and DNA was extracted and sequenced using the protocols described above. Genomes were assembled using the default parameters for de novo genome assembly within the CLC Workbench.

Conventional PCR. Seven candidate loci that met the criteria described above were tested with conventional PCR using a range of $F$. oxysporum isolates that were pathogenic or nonpathogenic on strawberry. Only those loci that were highly specific for pathogenic isolates with clear, single products were carried on in assay development. The PCR primers of the final locus used in this study are provided in Table 1 and were used at a concentration of $400 \mathrm{nM}$ in a $25 \mu \mathrm{l}$ reaction with $2 \mathrm{mM}$ of $\mathrm{MgCl}_{2}, 200 \mu \mathrm{M}$ of deoxyribonucleotide triphosphate (dNTPs) (New England Biolabs, Ipswich, MA), 1× AmpliTaq PCR buffer (Applied Biosystems, Foster City, CA), and 1 unit of AmpliTaq. The cycling parameters used to amplify the PCR product were one cycle 
of $95^{\circ} \mathrm{C}$ for $2 \mathrm{~min} ; 35$ cycles of $95^{\circ} \mathrm{C}$ for $30 \mathrm{~s}, 56^{\circ} \mathrm{C}$ for $30 \mathrm{~s}$, and $72^{\circ} \mathrm{C}$ for $30 \mathrm{~s}$; and a final extension cycle of $72^{\circ} \mathrm{C}$ for $5 \mathrm{~min}$.

The IGS rDNA region (primers NL11 and iNLR) and/or the $E F-1 \alpha$ gene (primers EF-3 and EF-22) were amplified from all DNA that was used for conventional PCR tests, recombinase polymerase amplification (RPA) assays, and quantitative polymerase chain reaction (qPCR) assays to ensure that the DNA was of sufficient quality for amplification (O'Donnell et al. 2009). Two nanograms of template DNA was used in these reactions. The $E F-1 \alpha$ gene was amplified using the same reaction mix and cycling parameters as described above except with an annealing temperature of $51^{\circ} \mathrm{C}$ and an extension time of $50 \mathrm{~s}$. The IGS rDNA region was amplified using the same reaction mix and cycling parameters described above except with a $53^{\circ} \mathrm{C}$ annealing temperature and a 1-min extension. PCR products were visualized on a 1 to $2 \%$ agarose gel to confirm that they were the correct size.

TaqMan qPCR. A TaqMan qPCR assay was developed with a multiplexed internal control that would indicate the presence of PCR inhibitors. The internal control was developed by Bilodeau et al. (2012) as a 121-bp PCR product created from a Pythium plasmid and the multiple cloning sites of pBluescript and pUC119 and was used at a dilution of $\sim 20 \mathrm{fg}$ so that the product would amplify at a $\mathrm{C}_{\mathrm{t}}$ around 31 to 32 in the final reaction. The internal control primer sequence for the $\mathrm{F}$ primer was $5^{\prime}$-CGTTTCCCGTTACTC TTCT- $3^{\prime}$ and the sequence for the R primer was $5^{\prime}$-GGATTTCGGC CCAGAAACT-3'. The probe used for the internal control was $5^{\prime}-\mathrm{Cal}$ Fluor Red 610-AAAGTAAGCTTATCGATACCGTCGACCTBHQ2-3' (Bilodeau et al. 2012). Reactions (25 $\mu \mathrm{l})$ were run with $400 \mathrm{nM}$ each of the $F$. oxysporum $\mathrm{f}$. sp. fragariae specific primers (Frag_F and R), $400 \mathrm{nM}$ each of the internal control $\mathrm{F}$ and $\mathrm{R}$ primers, $1 \times$ PerfeCTa Multiplex qPCR ToughMix (Quantabio, Beverly, MA), $200 \mathrm{nM}$ of the Frag_TaqMan_probe, $40 \mathrm{nM}$ of the internal control probe, and $20 \mathrm{fg}$ of the internal control (Table 1). The qPCR was run in a CFX96 Real-Time PCR Detection System (Bio-Rad Laboratories, Hercules, CA) with cycling parameters of one cycle of $95^{\circ} \mathrm{C}$ for $3 \mathrm{~min}$ and 50 cycles of $95^{\circ} \mathrm{C}$ for $15 \mathrm{~s}$ and $62^{\circ} \mathrm{C}$ for $30 \mathrm{~s}$ with a plate read. The baseline threshold for each fluorophore was set at 50 . Two nanograms of all DNA samples representing taxa listed in Table 2 was used to test the specificity of this assay and run in duplicate on the plate. An average of the two values was reported and used in calculations for each sample. Standard curves for this assay were produced using DNA prepared from three $F$. oxysporum f. sp. fragariae isolates in a dilution series from $50 \mathrm{fg}$ to $2 \mathrm{ng}$, and the average and standard deviation were calculated from these three biological replicates (Fig. 1).

As an additional test for some isolates, the Fofra locus from Suga et al. (2013) was used for development of a TaqMan qPCR amplification with an $\mathrm{F}$ primer of $5^{\prime}$-CAGACTGGGGTGCTTAAAGT-3', an R primer of '5'-AACCGCTAGGGTCGTAACAAA-3', and a probe of $5^{\prime}$-d-FAM-TAATGCATAACTAAAATTTCGCTCCTCC CATACA-BHQ1-3'. The same reagent concentrations, cycling parameters, and input DNA concentrations were used as previously described for the Frag locus, except the internal control primers and probes were not added to this reaction.

DNA extraction from soil samples. Soil samples collected from strawberry fields in Monterey and Ventura counties in California were mixed by hand, air dried for 2 weeks, and ground with an HM-375 soil grinder (Houghton Manufacturing, Vicksburg, MI) fitted with a No. 35 perforated plate and/or pulverized by hand in a mortar and pestle. For each soil sample, DNA from three replicates of 0.5-g samples was extracted using the FastDNA SPIN Kit for soil (MP Biomedicals, Santa Ana, CA) with some modifications. Samples were homogenized using a FastPrep FP120 homogenization instrument (MP Biomedicals) for $45 \mathrm{~s}$ at a speed of 6.5 (Bilodeau et al. 2012). After homogenization, the lysate was centrifuged at $16,000 \times g$ for $10 \mathrm{~min}$ to pellet soil and cellular debris. The supernatant was transferred to a new tube, the protein precipitation solution (PPS) was added, and the tube was inverted 20 times and centrifuged for $5 \mathrm{~min}$ at $16,000 \times g$. After centrifugation, the supernatant was removed and added to $800 \mu \mathrm{l}$ of binding matrix in a 2-ml Eppendorf tube. The binding matrix settled for 5 to $7 \mathrm{~min}$ after 2 min of inversions, and then $800 \mu \mathrm{l}$ of the supernatant was discarded. The matrix was resuspended in the remaining supernatant and applied to a SPIN filter where the matrix was washed and the DNA was eluted with $100 \mu \mathrm{l}$ of prewarmed DNase/pyrogen-free water (DES) buffer $\left(65^{\circ} \mathrm{C}\right)$. The elutant was then reapplied to the SPIN filter a second time to elute the DNA. Three microliters of DNA was used in all amplifications.

Development of the RPA assay and sample processing. Strawberry plants were processed for RPA by macerating $0.5 \mathrm{~g}$ of infected crown tissue with $5 \mathrm{ml}$ of $1 \times$ general extraction buffer 2 (GEB2)

Table 2. List of Fusarium oxysporum isolates that were used in this study and the results obtained from both the TaqMan quantitative polymerase chain reaction assay and the recombinase polymerase amplification assay

\begin{tabular}{|c|c|c|c|}
\hline Origin host & Location & Year $^{\mathbf{a}}$ & No. of isolates \\
\hline \multicolumn{4}{|c|}{ Positive isolate } \\
\hline Strawberry & California & 2014 & 6 \\
\hline Strawberry & California & 2015 & 5 \\
\hline Strawberry & Ventura County, CA & 2016 & 2 \\
\hline Strawberry & El Dorado County, CA & 2016 & 2 \\
\hline Strawberry & Ventura County, CA & 2017 & 10 \\
\hline Strawberry & Monterey County, CA & 2017 & 11 \\
\hline Strawberry & Santa Barbara County, CA & 2017 & 5 \\
\hline Strawberry & Santa Cruz County, CA & 2017 & 16 \\
\hline Strawberry & Japan & NA & 8 \\
\hline Strawberry & Australia & NA & 2 \\
\hline \multicolumn{4}{|c|}{ False-positive isolate } \\
\hline Strawberry & California & 2015 & 1 \\
\hline Strawberry & California & 2017 & 1 \\
\hline \multicolumn{4}{|c|}{ Negative isolate } \\
\hline Strawberry & California & 2015 & 5 \\
\hline Lettuce & California & 2016 & 3 \\
\hline Lettuce & Arizona & 2016 & 1 \\
\hline Strawberry & Santa Cruz County, CA & 2016 & 2 \\
\hline Strawberry & Monterey County, CA & 2017 & 1 \\
\hline Strawberry & Santa Cruz County, CA & 2017 & 5 \\
\hline Strawberry & Santa Barbara County, CA & 2017 & 2 \\
\hline Strawberry & California, Nursery & 2017 & 32 \\
\hline Lettuce & Arizona & 2017 & 4 \\
\hline Strawberry & Ventura County, CA & 2017 & 2 \\
\hline Strawberry & Australia & NA & 6 \\
\hline Various & Worldwide & NA & 44 \\
\hline
\end{tabular}

a NA $=$ multiple years are represented or the year is unknown.

Table 1. Primers and probe sequences that were used to detect Fusarium oxysporum f. sp. fragariae

\begin{tabular}{|c|c|c|c|}
\hline Primer/probe name ${ }^{\mathbf{a}}$ & Primer sequence & Length (nt) & GC $(\%)$ \\
\hline Frag_F & GTGAGACGGACATCTTGAAG & 20 & 50 \\
\hline Frag_R & AGGAACATTTCCAGCACCGA & 20 & 53 \\
\hline Frag_TaqMan_probe & FAM-CACTTGCCTTTGGGCTCTGGATACTAAC-BHQ1 & 28 & 50 \\
\hline Frag_RPA_F & GCCATGCGCATCCCAAGTGAGACGGACATCTTGA & 34 & 56 \\
\hline Frag_RPA_R & GCATAGTACAAGGAACATTTCCAGCACCGAAAC & 33 & 45 \\
\hline Frag_RPA_probe & $\begin{array}{l}\text { TGATGCTGCCACTTGCCTTTGGGCTCTGGA[T(FAM)][dSpacer]C } \\
\text { [T(BHQ-1)]AACTCCAGCAGCAATTCG-C3 spacer }\end{array}$ & 51 & 53 \\
\hline
\end{tabular}

${ }^{\mathrm{a}} \mathrm{F}=$ forward, and $\mathrm{R}=$ reverse. 
(Agdia Inc., Elkhart, IN) (Miles et al. 2015). One microliter of the extract was used immediately or the extract was flash-frozen and stored at $-80^{\circ} \mathrm{C}$ for future use. The assay was also tested with $2 \mathrm{ng}$ of DNA purified as described above from a pure culture of the fungus. The standard curve was created by adding $1 \mu l$ of purified DNA to $1 \mu 1$ of GEB2-based crown extract from an uninfected plant.

The RPA assay was developed using the same locus that was used for the TaqMan qPCR assay and with the primers and probes shown in Table 1. The coxl gene-based plant internal control was used with the F primer sequence, Cox1_IPC_RPA_F (5'-CATGCGTGGAC CTGGAATGACTATGCATAGA-3') and the R primer sequence, Cox1_IPC_RPA_R (5'-GGTTGTATTAAAGTTTCGATCGGTTA ATAACA-3' (Miles et al. 2015). The probe for the plant internal control was GGTCCGTTCTAGTGACAGCATTCCYACTTTTATTA [CAL Fluor Orange 560-dT] C [THF] C [BHQ1-dT] YCCGGTA CTGGC [3'-C3SPACER] (Miles et al. 2015). The TwistAmp exo kit (TwistDx, Cambridge, UK) was used with a final concentration of $300 \mathrm{nM}$ of the Frag_RPA_F and Frag_RPA_R primers, $250 \mathrm{nM}$ of the Cox1_IPC_RPA_F and Cox1_IPC_RPA_R primers, $120 \mathrm{nM}$ of each probe, $14 \mathrm{mM}$ of magnesium acetate, and $29.5 \mu \mathrm{l}$ of the TwistAmp exo rehydration buffer in a 50- $\mu$ l reaction. The reaction was prepared in a PCR strip tube with the master mix (except for magnesium acetate) and the sample and was transferred to the TwistDx reaction tubes with a multichannel pipette. The liquid was mixed with the lyophilized reaction pellet within the TwistDx tubes by pipetting up and down 12 times. A single TwistDx microball was added to each tube to facilitate mixing during amplification. Magnesium acetate $(2.5 \mu \mathrm{l})$ was added to the inside of the lids of the strip tube caps and mixed with the rest of the reagents via a short centrifugation step immediately prior to starting the instrument reading. The reaction was run using an Axxin T16-ISO instrument (Fairfield, NSW, Australia) with version 1.8.0.3 software with the fluorescence channels set at 7\% FAM, $40 \%$ Hex, and $25 \%$ Rox. The instrument was set at $39^{\circ} \mathrm{C}$ for 30 cycles. with each cycle consisting of a 50-s read period and a 10-s pause period. Data were analyzed by exporting readings as an Excel file and calculating the slope as a change in fluorescence signal over a time period between 5 and $25 \mathrm{~min}$. The cutoff slope values used to determine a positive result were 12 for the plant internal control and six for $F$. oxysporum $\mathrm{f}$. sp. fragariae. The onset of amplification was identified as the time (in seconds) at which the fluorescence value crossed 500. The log of this value was used to create the standard curves to determine the sensitivity for this assay (Fig. 2).

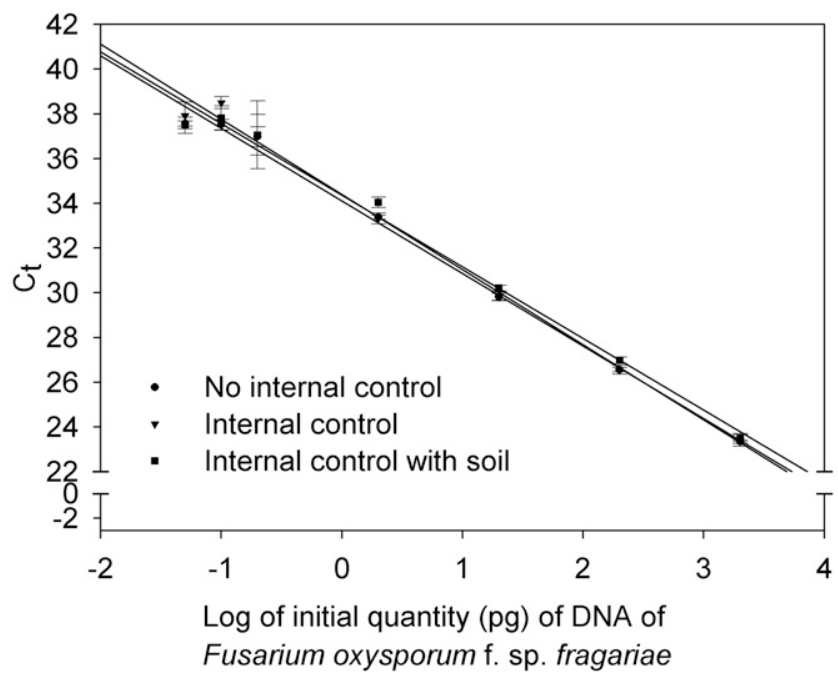

Fig. 1. TaqMan assay using a serial dilution of purified Fusarium oxysporum f. sp. fragariae DNA from $2 \mathrm{ng}$ to $50 \mathrm{fg}$ with an internal control and/or soil extract. Three biological replicates of each serial dilution were tested with two technical replicates on each plate that were averaged to give a single value for each biological replicate. Error bars represent the standard deviation from three biological replicates. Only two biological replicates were detected for some samples of a concentration of $100 \mathrm{fg}$ or less. $C_{t}=$ cycle threshold.
Pathogenicity test setup and statistical evaluation of the isolates. Isolates used for pathogenicity tests were first single spored or hyphal tipped to ensure that a single isolate was evaluated. These isolates were grown on 8 to 10 Petri plates of potato dextrose agar for 8 to 10 days under continuous light at room temperature. Conidia were suspended by pouring 3 to $4 \mathrm{ml}$ of sterile water per plate and gently dislodging conidia with a plate spreader. Conidial suspensions were kept chilled while the spores were counted with a hemocytometer and diluted with water to a final concentration of $1 \times 10^{5}$ spores $/ \mathrm{ml}$. Eight Albion strawberry crowns per treatment were washed and the roots were trimmed to a final length of 3 to 4 inches. The crowns were placed in the conidial suspension for $1 \mathrm{~h}$ prior to potting in a sterilized mixture of sandy soil. Every experiment included water as a negative control and the isolate 15-2 was used as a positive control.

Inoculated plants were placed in a greenhouse and bottom watered as needed. The inoculated plants were rated from 6 to 10 weeks after inoculation. The rating scale was the following: $0=$ healthy plant; $1=$ a leaf or two displaying wilting and/or moderate stunting; 2 = many leaves wilted, severe stunting, or moderate wilting and stunting; and $3=$ dead plant. The ratings were analyzed using the Kruskal-Wallis test in R software followed by pairwise comparisons using the Wilcoxon rank-sum test. Isolates with an adjusted $P$ value (Benjamini and Hochberg 1995) of $<0.05$ when compared with the water control were considered to be pathogenic. Crowns and/or petioles of infected plants were frequently cultured to confirm the presence of the Fusarium pathogen. Tissue was dipped for $10 \mathrm{~s}$ in $70 \%$ ethanol, followed by immersion in $1 \%$ sodium hypochlorite for $1 \mathrm{~min}$ and a rinse with sterile water. Plant pieces were placed on potato dextrose agar and incubated at 25 to $28^{\circ} \mathrm{C}$ for 2 to 4 days until the Fusarium pathogen was observed growing from the positive control.

\section{Results}

Identification of a locus unique to $F$. oxysporum $\mathrm{f}$. sp. fragariae using comparative genomics. The unique approach of using two workflows within CLC Workbench enabled efficient identification of candidate loci for detection. The first workflow set strict thresholds to eliminate any regions where reads mapped from off-target isolates. Using this approach, more than 250 candidate loci were identified that were completely absent in the nontarget isolates evaluated. The second workflow used sequence data from target isolates to ensure that candidate loci were present in entirety in the genomes of multiple isolates of $F$. oxysporum $\mathrm{f}$. $\mathrm{sp}$. fragariae. Fewer than 100 candidates remained after completion of the second workflow. Additional BLAST and GC content analyses further narrowed the list of candidates before selection of loci suitable for RPA probe design,

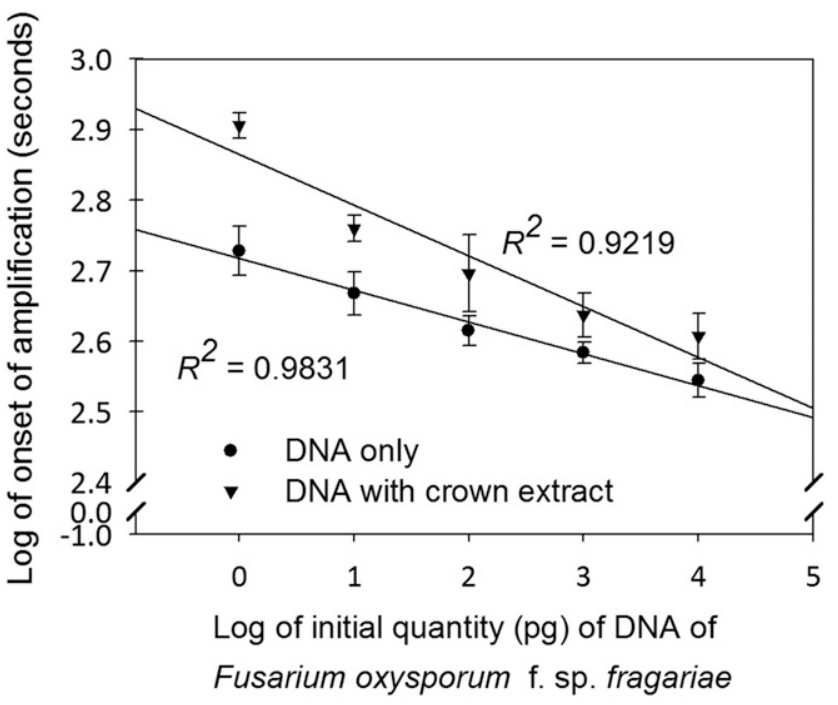

Fig. 2. Onset of amplification of the recombinase polymerase amplification assay using a serial dilution of purified DNA from Fusarium oxysporum f. sp. fragariae 10 $\mathrm{ng}$ to $1 \mathrm{pg}$ with and without a crude strawberry crown extract. Error bars represent the standard deviation from three biological replicates. 
which has specific requirements for length and location of $\mathrm{T}$ nucleotides for modifications. Primers were designed for seven candidates and were tested using conventional PCR against a larger panel of isolates representing $F$. oxysporum isolates that were pathogenic and nonpathogenic on strawberry (Table 2). From this initial testing, a final single-copy candidate locus in the core genome was chosen (Supplementary Fig. S1) that showed robust, single-target amplification in pathogenic isolates and no off-target amplification in nonpathogenic isolates.

Development of a TaqMan assay. A TaqMan qPCR assay was developed that detected $F$. oxysporum $\mathrm{f}$. sp. fragariae using DNA from pure fungal cultures or from soil samples. The sensitivity of this assay was evaluated using three isolates of $F$. oxysporum f. sp. fragariae, which were tested from 2 ng to 50 fg using two technical replicates per sample on the same plate that were then averaged to give a value for each DNA sample from each isolate. These three values were then averaged and the mean and standard deviations are shown on the standard curve in Figure 1. Some replicates for the lowest amounts of DNA, 100 and $50 \mathrm{fg}$, did not consistently amplify, but the 200-fg sample amplified in all replicates in all of the situations tested. Using an internal control when running a qPCR assay with soil samples is important to monitor for the presence of inhibitors; therefore, the purified DNA samples were tested in the presence of an internal control and in the presence of $3 \mu \mathrm{l}$ of DNA extracted from a soil sample free of the pathogen to evaluate the culture-based DNA detection limit and efficiency of the assay in the presence of soil extract. The presence of the internal control and/or the soil extract did not affect the sensitivity of the assay; in all cases, the amplification efficiency was between 96 and 102\% (Fig. 1).

Specificity of the qPCR assay was tested using a range of more than 190 DNA samples collected from strawberry plants in California and from other hosts, states, and countries. Of the DNAs that were tested with this assay, 67 were true positives and 116 were true negatives. Many of these isolates, especially those from California strawberry plants, have been evaluated with pathogenicity tests and many of the negative isolates were received from well-established culture collections that were sourced from nonstrawberry hosts (Table 2). Nine isolates tested negative that were other pathogen species commonly associated with strawberry, including Phytophthora cactorum, $P$. fragariae, Verticillium dahliae, and Macrophomina phaseolina. Two false-positive $F$. oxysporum isolates, both

Table 3. Results of the strawberry crown samples that were tested using the recombinase polymerase amplification (RPA) assay

\begin{tabular}{lccc}
\hline Location & RPA $^{\mathbf{a}}$ & Year & No. of samples \\
\hline True-positive sample & & & \\
$\quad$ Ventura County, CA & + & 2016 & 6 \\
El Dorado County, CA & + & 2016 & 4 \\
Santa Cruz County, CA & + & 2016 & 1 \\
Monterey County, CA & + & 2016 & 2 \\
California, unknown county & + & 2016 & 1 \\
Ventura County, CA & + & 2017 & 17 \\
Monterey County, CA & + & 2017 & 11 \\
Santa Barbara County, CA & + & 2017 & 2 \\
Santa Cruz County, CA & + & 2017 & 9 \\
California, unknown county & + & 2017 & 4 \\
True-negative sample & & & \\
Ventura County, CA & - & 2016 & 5 \\
Monterey County, CA & - & 2016 & 4 \\
Santa Cruz County, CA & - & 2016 & 2 \\
Monterey County, CA & - & 2017 & 2 \\
Santa Cruz County, CA & - & 2017 & 5 \\
Santa Barbara County, CA & - & 2017 & 1 \\
California, nursery & - & 2017 & 39 \\
California, unknown county & - & 2017 & 3 \\
False-negative sample & & & \\
Santa Cruz County, CA & - & 2017 & 1 \\
Santa Barbara County, CA & - & 2017 & 1 \\
\hline
\end{tabular}

${ }^{a}$ Plus signs indicate positive results, and minus signs indicate negative results. recovered from strawberry but found to be nonpathogenic, were detected and the diagnostic specificity of the assay was determined to be $98.1 \%$. No false negatives were detected, and the sensitivity was $100 \%$.

Soil samples collected from production fields with a history of Fusarium wilt were processed and the DNA was run with the TaqMan qPCR as a proof of concept for using the TaqMan assay for soil samples. Six soils with known populations of $F$. oxysporum f. sp. fragariae had all three soil extraction replicates amplify in the qPCR assay, with the lowest count being 20 to $50 \mathrm{CFU} / \mathrm{g}$, based on soil dilution plating as described by Henry et al. (2017). Three additional soils with levels of $F$. oxysporum $\mathrm{f}$. sp. fragariae below 50 $\mathrm{CFU} / \mathrm{g}$ did not consistently amplify with this assay. Likewise, there was no amplification from several other soil samples that did not have detectable levels of $F$. oxysporum $\mathrm{f}$. sp. fragariae in soil plate counts, which indicates that this assay does not nonspecifically amplify common microorganisms in the soil.

Development of an RPA assay. An RPA assay was developed for the specific detection of $F$. oxysporum $\mathrm{f}$. sp. fragariae and tested extensively with DNA extracted from pure cultures and plant samples. The RPA assay was designed using the primers and probes in Table 1 . Three different $F$. oxysporum $\mathrm{f}$. sp. fragariae isolates were tested from $10 \mathrm{ng}$ to $1 \mathrm{pg}$ with and without the presence of strawberry crown extract and the detection limit was found to be $1 \mathrm{pg}$ (Fig. 2). The presence of the crown extract increased the time to the onset of amplification but did not affect the final detection limit of the assay with $1 \mathrm{pg}$ of DNA amplifying before $10 \mathrm{~min}$ without crown extract and before $15 \mathrm{~min}$ in the presence of crown extract (Fig. 2). The specificity of the RPA assay was tested with more than $180 \mathrm{~F}$. oxysporum isolates from California and around the world, with hosts ranging from strawberry to banana (Table 2). Of the 67 isolates that were true-positive samples, 57 were from California, with 45 isolates confirmed as $F$. oxysporum $\mathrm{f}$. sp. fragariae by pathogenicity tests. Of the 116 isolates that tested negative with RPA, 52 were from California, including more than 30 that were collected from strawberry nursery plants. Other isolates that were considered positive were collected from plants with symptoms and cultured $F$. oxysporum that were consistent with the isolates that were confirmed with the pathogenicity tests; furthermore, many of these isolates also tested positive with the Suga locus (Suga et al. 2013). More than 50 of the negative isolates included formae speciales that were collected from nonstrawberry hosts, including soybean, tomato, sweet potato, corn, spinach, lettuce, and celery. Other samples that tested negative were pathogens commonly associated with strawberry, including $P$. cactorum, $P$. fragariae, V. dahliae, and $M$. phaseolina. The diagnostic sensitivity of this assay was $100 \%$ with the isolates tested. In addition, there were two false positives from this assay, which were isolates of $F$. oxysporum that were collected from strawberry plants but were found to be nonpathogenic (the same false positives observed in the TaqMan assay). The overall diagnostic specificity for this assay using DNA was calculated to be $98.1 \%$.

Validation of the RPA assay with infected plant samples. To test the efficacy of the RPA assay in potential field settings, 120 different strawberry crowns from California fruit production and nursery fields were tested with the RPA assay. All tested plants were showing symptoms associated with Fusarium wilt and/or had positive culture results indicating that $F$. oxysporum species complex isolates were present in plated plant tissue. Of these, 57 tested positive and 61 were negative for $F$. oxysporum f. sp. fragariae (Table 3 ). Two were false negatives, as isolates that were cultured from these plants were later found to be pathogenic (Tables 3 and 4).

In addition to the large number of strawberry plants that were tested with the RPA assay alone, a subsample of 50 strawberry crown samples was evaluated with several types of tests to ensure that the RPA was able to specifically detect as positive plants that were infected with $F$. oxysporum f. sp. fragariae and not those that had been improperly diagnosed as having a Fusarium infection from common symptoms or from the recovery of a Fusarium isolate that was a result of a secondary colonization (Table 4). To do this, each sample that was tested with RPA was also cultured and the resulting fungal 
cultures were tested with the qPCR assay that was developed within this study and with the qPCR assay that was developed with the primers published by Suga et al. (2013). The pathogenicity of each isolate was also tested using at least seven crowns of the highly susceptible cultivar, Albion (Henry et al. 2017), per pathogenicity test replicate.

The primers developed by Suga et al. (2013) served as a check of the qPCR assay developed in this study and helped to corroborate positive or negative results by using a secondary locus. In four cases, the Suga-based qPCR result was negative and qPCR using the same DNA tested positive with the qPCR assay developed in this study (Table 4). Three of these isolates tested positive in subsequent pathogenicity tests, thus highlighting that the locus developed in this study can detect all strains of $F$. oxysporum $\mathrm{f}$. sp. fragariae found in California, whereas the Suga locus does not (Table 4). In one case, the Suga marker tested negative and the isolate was nonpathogenic, but the marker developed in this study tested positive. Two of the isolates that were negative for the Suga marker and positive in the TaqMan assay in this study were recovered from plants that tested negative with the RPA assay (Table 4). The one isolate that gave a false positive with the TaqMan assay in this study was recovered from a plant that tested negative with RPA. In a few cases, multiple small crowns from a single site were collected and separate crowns had to be used for RPA and plating because of limitations in the amount of tissue available, which also could have led to discrepancies between the plant and culture results. It is also possible that the level of $F$. oxysporum $\mathrm{f}$. sp. fragariae present within the crown tissue used for RPA was below the detection limit of the assay.

Given these exceptions, the rest of the strawberry crowns tested yielded consistent results between the RPA test of the crown tissue, the qPCR assays of the DNA extracted from the isolate recovered from the plant, and the pathogenicity tests using that same isolate (Table 4). In many cases, the pathogenicity tests were completed with two biological replicates of seven plants each. Overall, the RPA assay with crown tissue had a diagnostic sensitivity of $92.3 \%$ and a diagnostic specificity of $100 \%$ (Table 3 ).

\section{Discussion}

Given the polyphyletic relationships of isolates from formae speciales within the $F$. oxysporum species complex, it was important to find a unique locus for isolates of $F$. oxysporum that were pathogenic on strawberry. The asexual nature of the pathogen and the ability of
F. oxysporum to horizontally transfer genes related to host pathogenicity meant that genes typically used for classification, including $I G S$, internal transcribed spacer (ITS), and EFla did not have sufficient genetic information to distinguish pathogens from nonpathogens of strawberry (A. Burkhardt and F. Martin, unpublished data; Henry et al. 2017; Michielse and Rep 2009; O'Donnell et al. 2009). As a result, a novel approach using whole genome sequencing and comparative genomics was used in this work to identify a unique genetic region associated with $F$. oxysporum $\mathrm{f}$. sp. fragariae. Initial work to carefully select a range of $F$. oxysporum isolates collected from strawberry that were from different SCGs and were pathogenic and not pathogenic enabled a diverse data set to be compared using the fewest number of isolates (Henry et al. 2017). It was especially important that all of the SCGs were represented, given that the assay developed by Suga et al. (2013) did not detect $F$. oxysporum f. sp. fragariae within all of the SCGs found within California (Henry et al. 2017). A dual workflow within CLC Workbench allowed for the next-generation sequencing from the total genome of each isolate to be compared efficiently to develop a list of present/absent candidate loci that are ideal for developing TaqMan and RPA assays.

A challenge in developing these assays was to ensure that the assays only detected pathogenic $F$. oxysporum isolates recovered from strawberry. Many isolates collected from this host were not pathogenic but had IGS and EFI $a$ sequences similar or identical to pathogenic isolates, which made determining the specificity and sensitivity of this assay difficult. To confirm that the results of the developed assays were accurate, pathogenicity tests were performed on 50 isolates that were recovered from strawberry plants. Although the results of these assays are consistent across multiple diagnostic platforms and with multiple markers, it is still possible that some of the isolates that are considered as nonpathogenic could be pathogenic under different growing conditions or when infecting different strawberry varieties (Paynter et al. 2016). Furthermore, some nonpathogenic isolates thought to be $F$. oxysporum were later sequenced and determined to be $F$. proliferatum based on nearly identical regions of the EF-1 $\alpha$ gene (JX869031.1) (Masratul Hawa et al. 2013) in the sequenced genome of these isolates. When inoculated onto strawberry plants, isolates of $F$. proliferatum did not induce symptoms that were significantly different from those of the water-treated control in terms of the wilting and stunting rating scale used in this study. However, future work is needed to investigate the role that $F$. proliferatum might play in infecting strawberry and the full range of potential

Table 4. Strawberry plant samples tested with the recombinase polymerase amplification (RPA) assay and the corresponding quantitative polymerase chain reaction (qPCR) and pathogenicity test results of the cultures isolated from each sample ${ }^{\mathrm{a}}$

\begin{tabular}{|c|c|c|c|c|c|c|}
\hline Location & Year & Plant RPA results & Culture qPCR & Suga qPCR & Pathology test results & No. of samples \\
\hline Ventura County, CA & 2016 & + & + & + & + & 2 \\
\hline El Dorado County, CA & 2016 & + & + & + & + & 2 \\
\hline Santa Cruz County, CA & 2016 & - & - & - & - & 2 \\
\hline Ventura County, CA & 2017 & + & + & + & + & 2 \\
\hline Monterey County, CA & 2017 & + & + & + & + & 6 \\
\hline Monterey County, CA & 2017 & - & - & - & - & 1 \\
\hline Santa Cruz County, CA & 2017 & - & - & - & - & 2 \\
\hline Santa Barbara County, CA & 2017 & - & - & - & - & 1 \\
\hline Santa Barbara County, CA & 2017 & + & + & + & + & 1 \\
\hline Santa Cruz County, CA & 2017 & + & + & + & + & 2 \\
\hline Santa Cruz County, CA & 2017 & - & + & - & + & 1 \\
\hline Santa Barbara County, CA & 2017 & + & + & + & $t^{\mathrm{b}}$ & 1 \\
\hline Santa Barbara County, CA & 2017 & - & + & - & + & 1 \\
\hline Monterey County, CA & 2017 & + & + & - & + & 1 \\
\hline Santa Cruz County, CA & 2017 & + & + & + & + & 3 \\
\hline California nursery & 2017 & - & - & - & - & 18 \\
\hline California nursery & 2017 & - & + & - & $-^{\mathrm{b}}$ & 1 \\
\hline Monterey County, CA & 2017 & + & + & + & $-\mathrm{b} /+^{\mathrm{b}}$ & 1 \\
\hline Ventura County, CA & 2017 & + & + & + & + & 2 \\
\hline
\end{tabular}

${ }^{a}$ All pathogenicity tests were completed with two replicates unless otherwise indicated. Plus signs indicate positive results, and minus signs indicate negative results.

${ }^{\mathrm{b}}$ One pathogenicity test replicate was completed for the isolate from this sample. 
pathogenicity in many of the isolates tested. Nevertheless, typical growing conditions and a known susceptible cultivar of strawberry were used to conduct the pathogenicity tests, and the likelihood that any of the isolates that are not detected in this assay are aggressive pathogens of strawberry is very low (Fang et al. 2012; Henry et al. 2017).

Overall, the work described in this study offers an improved diagnostic locus, especially for $F$. oxysporum f. sp. fragariae isolates from California. Perfect (100\%) diagnostic sensitivity was achieved with both the TaqMan qPCR assay and the RPA assay with the Californian isolates that were evaluated. This is improved over the lower sensitivity (93\%) for the California isolates that was reported when using the Fofra locus of Suga et al. (2013) for pathogen detection (Henry et al. 2017). Specifically, there were five pathogenic California isolates that tested positive with the assays developed in this study that were negative with the TaqMan qPCR assay using the Suga locus that was developed as part of this project. Hong et al. (2018) developed a SYBR Green assay using a portion of the Suga locus; however, because of a limited number of taxa that it was validated against and their amplicon not including the unique 5 -terminal $\sim 40 \mathrm{bp}$ of the locus (leaving the $5^{\prime}$-end of their locus with 95 to $97 \%$ sequence similarity to other $F$. oxysporum formae speciales in our database), additional testing with California isolates would be needed to confirm specificity.

The specificity of the TaqMan and RPA assays with DNA isolated from fungal cultures was $98.1 \%$. A number of formae speciales and other common strawberry pathogens tested negative, indicating that these assays did not have any off-target detection. Of the two isolates that did give a false-positive result, one of them was sequenced with Illumina and the data indicated that it grouped within the main SCGs of F. oxysporum f. sp. fragariae (F. Martin, D. Geiser, and P. Henry, personal communication) and had a full copy of the detection locus. It may be possible that these isolates have lost the ability to infect strawberry or may be pathogenic on cultivars of strawberry other than the one that was used in the pathogenicity tests. When the RPA assay was tested on plants from strawberry production fields and nurseries, a diagnostic sensitivity of $92.3 \%$ and a diagnostic specificity of $100 \%$ was observed, with the sensitivity likely improved by better sampling of infected tissue in the future. Overall, this assay works very well for $F$. oxysporum $\mathrm{f}$. sp. fragariae isolates from California.

Our preliminary work testing this locus on international isolates, especially those from Australia and Japan, shows that it has promise for identifying this pathogen in other countries. In total, 46 international isolates of $F$. oxysporum were tested, with 31 of them being true negatives, many of which represented other formae speciales. Of 21 isolates tested from Japan and Australia, 10 were true positives, six were true negatives, and five were false negatives (one from Japan and four from Australia). However, the false negatives from Australia did not cause leaf chlorosis, which is a symptom caused by all other isolates of $F$. oxysporum $\mathrm{f}$. sp. fragariae that were tested for pathogenicity. Preliminary phylogenetic analysis suggests that these Australian isolates do not cluster with the other $F$. oxysporum f. sp. fragariae isolates tested (P. Henry and T. Gordon, F. Martin, and D. Geiser, personal communication). A more comprehensive phylogenetic analysis and examination of symptom type is needed to clarify the evolutionary relationships within $F$. oxysporum $\mathrm{f}$. $\mathrm{sp}$. fragariae and to determine whether the isolates causing this symptom type are reflective of a distinct group of pathogenic isolates.

The TaqMan assay developed in this study has the potential for use in quantifying $F$. oxysporum f. sp. fragariae in soil. Currently, this assay can detect moderate levels of $F$. oxysporum $\mathrm{f}$. sp. fragariae from DNA extracted from $0.5 \mathrm{~g}$ of soil, but additional soils with a range of inoculum densities need to be evaluated and extraction protocols should be developed to use a higher input level of soil to improve sensitivity for quantification of lower levels of inoculum in field soil. Additional studies should also be done to correlate the colony-forming units per gram of soil plating counts to the $C_{t}$ values from the qPCR assays so that this assay can be used for quantification in addition to the high-level qualitative testing for which it is now appropriate. Until then, the TaqMan assay can be used as a qualitative assay to determine whether $F$. oxysporum $\mathrm{f}$. sp. fragariae is present in the soil, and the developed RPA assay can be used for in-field diagnostics or rapid diagnostics in a clinic setting that will facilitate implementation of improved disease management practices.

\section{Acknowledgments}

Mention of trade names or commercial products in this article is solely for providing specific information and does not imply recommendation or endorsement by the U.S. Department of Agriculture. We thank K. O'Donnell and D. Geiser for providing some of the DNAs of other formae speciales for validating specificity and N. Luecke, S. Cude, A. Shabazian, and C. Coelho for help with the pathogenicity tests.

\section{Literature Cited}

Altschul, S. F., Gish, W., Miller, W., Myers, E. W., and Lipman, D. J. 1990. Basic local alignment search tool. J. Mol. Biol. 215:403-410.

Benjamini, Y., and Hochberg, Y. 1995. Controlling the false discovery rate: A practical and powerful approach to multiple testing. J. R. Stat. Soc. B 57: 289-300.

Bilodeau, G. J., Koike, S. T., Uribe, P., and Martin, F. N. 2012. Development of an assay for rapid detection and quantification of Verticillium dahliae in soil Phytopathology 102:331-343.

Bilodeau, G. J., Martin, F. N., Coffey, M. D., and Blomquist, C. L. 2014 Development of a multiplex assay for genus- and species-specific detection of Phytophthora based on differences in mitochondrial gene order. Phytopathology 104:733-748.

Fang, X., Phillips, D., Verheyen, G., Li, H., Sivasithamparam, K., and Barbetti, M J. 2012. Yields and resistance of strawberry cultivars to crown and root diseases in the field, and cultivar responses to pathogens under controlled environment conditions. Phytopathol. Mediterr. 51:69-84.

Geiser, D. M., Gasco-Jimenez, M. del M., Kang, S., Makalowska, I., Veeraraghavan, N., Ward, T. J., Zhang, N., Kuldau, G. A., and O'Donnell, K. 2004. FUSARIUM-ID v. 1. 0: A DNA sequence database for identifying Fusarium. Eur. J. Plant Pathol. 110:473-479.

Gordon, T. R. 2017. Fusarium oxysporum and the Fusarium wilt syndrome. Annu. Rev. Phytopathol. 55:23-39.

Henry, P. M., Kirkpatrick, S. C., Islas, C. M., Pastrana, A. M., Yoshisato, J. A., and Pathology, P. 2017. The population of Fusarium oxysporum f. sp. fragariae, cause of Fusarium wilt of strawberry, in California. Plant Dis. 1:1-7.

Hong, S. W., Kim, D.-R., Kim, J. S., Cho, G., Jeon, C. W., and Kwak, Y.-S. 2018. Development qRT-PCR protocol to predict strawberry Fusarium wilt occurrence. Plant Pathol. J. 34:163-170.

Koike, S. T., and Gordon, T. R. 2015. Management of Fusarium wilt of strawberry. Crop Prot. 73:67-72.

Koike, S. T., Kirkpatrick, S. C., and Gordon, T. R. 2009. Fusarium wilt of strawberry caused by Fusarium oxysporum in California. Plant Dis. 93:1077.

Ma, L.-J., Charlotte Van Der Does, H., Borkovich, K. A., Coleman, J. J., Daboussi, M.-J., Di Pietro, A., Dufresne, M., Freitag, M., Grabherr, M., Henrissat, B., Houterman, P. M., Kang, S., Shim, W.-B., Woloshuk, C., Xie, X., Xu, J.-R., Antoniw, J., Baker, S. E., Bluhm, B. H., Breakspear, A., Brown, D. W., Butchko, R. A. E., Chapmen, S., Coulson, R., Coutinho, P. M., Danchin, E. G. J., Diener, A., Gale, L. R., Gardiner, D. M., Goff, S., HammondKosack, K. E., Hilburn, K., Hua-Van, A., Jonkers, W., Kazan, K., Kodira, C. D., Koehrsen, M., Kumar, L., Lee, Y.-H., Li, L., Manners, J. M., MirandaSaavedra, D., Mukherjee, M., Park, G., Park, J., Park, S.-Y., Proctor, R. H., Regev, A., Ruiz-Roldan, M. C., Sain, D., Sakthikumar, S., Sykes, S., Schwartz, D. C., Turgeon, B. G., Wpainski, I., Yoder, O., Young, S., Zeng, Q., Zhou, S., Galagan, J., Cuomo, C. A., Kistler, H. C., and Rep, M. 2010 Comparative genomics reveals mobile pathogenicity chromosomes in Fusarium. Nature 464:367-373.

Ma, L.-J., Geiser, D. M., Proctor, R. H., Rooney, A. P., O’Donnell, K., Trail, F., Gardiner, D. M., Manners, J. M., and Kazan, K. 2013. Fusarium pathogenomics. Annu. Rev. Microbiol. 67:399-416.

Masratul Hawa, M., Salleh, B., and Latiffah, Z. 2013. Characterization and pathogenicity of Fusarium proliferatum causing stem rot of Hylocereus polyrhizus in Malaysia. Ann. Appl. Biol. 163:269-280.

Michielse, C. B., and Rep, M. 2009. Pathogen profile update: Fusarium oxysporum. Mol. Plant Pathol. 10:311-324.

Miles, T. D., Martin, F. N., and Coffey, M. D. 2015. Development of rapid isothermal amplification assays for detection of Phytophthora species in plant tissue. Phytopathology 105:265-278

O'Donnell, K., Gueidan, C., Sink, S., Johnston, P. R., Crous, P. W., Glenn, A., Riley, R., Zitomer, N. C., Colyer, P., Waalwijk, C., van der Lee, T., Moretti, A., Kang, S., Kim, H.-S., Geiser, D. M., Juba, J. H., Baayen, R. P., Cromey, M. G., Bithell, S., Sutton, D. A., Skovgaard, K., Ploetz, R., Kistler, H. C., Elliott, M., Davis, M., and Sarver, B. A. J. 2009. A two-locus DNA sequence database for typing plant and human pathogens within the Fusarium oxysporum species complex. Fungal Genet. Biol. 46:936-948.

Paynter, M. L., Czislowski, E., Herrington, M. E., and Aitken, E. A. B. 2016 Differences in pathogenicity, genetic variability and cultivar responses among 
isolates of Fusarium oxysporum from strawberry in Australia. J. Am. Soc. Hortic. Sci. 141:645-652.

Pincot, D. D. A., Poorten, T. J., Hardigan, M. A., Harshman, J. M., Acharya, C. B., Cole, G. S., Gordon, T. R., Stueven, M., Edger, P. P., and Knapp, S. J. 2018. Genome-wide association mapping uncovers $F w 1$, a dominant gene conferring resistance to Fusarium wilt in strawberry. G3 (Bethesda) 8: 1817-1828.

Suga, H., Hirayama, Y., Morishima, M., and Suzuki, T. 2013. Development of PCR primers to identify Fusarium oxysporum f. sp. fragariae. Plant Dis. 97: 619-625. van Dam, P., de Sain, M., ter Horst, A., van der Gragt, M., and Rep, M. 2018. Use of comparative genomics-based markers for discrimination of host specificity in Fusarium oxysporum. Appl. Environ. Microbiol. 84:e01868-17.

van Dam, P., Fokkens, L., Ayukawa, Y., van der Gragt, M., ter Horst, A., Brankovics, B., Houterman, P. M., Arie, T., and Rep, M. 2017. A mobile pathogenicity chromosome in Fusarium oxysporum for infection of multiple cucurbit species. Sci. Rep. 7:9042.

van Dam, P., Fokkens, L., Schmidt, S. M., Linmans, J. H. J., Kistler, H. C., Ma, L. J., and Rep, M. 2016. Effector profiles distinguish formae speciales of Fusarium oxysporum. Environ. Microbiol. 18:4087-4102. 